\title{
EDUCACIÓN
}

\section{Despertar de las vocaciones científicas, desafíos y oportunidades}

\author{
Norma Edith López Rolandi ${ }^{1}$
}

\begin{abstract}
Resumen
Introducción: La mejora de logros de aprendizajes en ciencias naturales es una materia pendiente en Latinoamérica. Más del $80 \%$ de los estudiantes de 15 países investigados no han superado las pruebas de ciencias naturales en los cinco ejes temáticos analizados; salud, seres vivos, ambiente, tierra y el sistema solar, materia y energía, así como tampoco, se aplica el pensamiento crítico como un mecanismo para resolver problemas de contextos cotidianos.

El estilo de enseñanza que tiene cada docente es un rasgo individual que lo caracteriza de forma estable y general. Tomando esta premisa, y a la luz de varias investigaciones, se puede afirmar que la enseñanza de las ciencias en el sistema escolar de Paraguay, se desarrolla bajo un modelo tradicional.

A la luz de los nuevos desafíos del siglo XXI es necesario robustecer el curriculum de formación docente para una educación en ciencias de calidad, relevante y sustantiva.
\end{abstract}

Objetivo: El objetivo de esta investigación fue realizar la revisión bibliográfica de los modelos tradicionales de enseñanza de las ciencias naturales y cooperar en la difusión de las propuestas de mejoras ya realizadas en otras investigaciones. Material y Método: En esta investigación se sistematiza e integran resultados de investigaciones publicadas sobre los retos de cara a los magros logros de aprendizajes obtenidos en ciencias, por los estudiantes de la región y específicamente los de Paraguay.

Dadas las dificultades de los docentes en entender cómo aplicar eficientemente la didáctica en la enseñanza de las ciencias, con esta revisión se ha organizado la información empírica, con el objeto de contrastar y encontrar convergencias entre los diversos modelos, que permita trazar un recorrido de oportunidades para la mejora del modelo de formación y la práctica docente en Paraguay donde se evidencian brechas importantes en el conocimiento de los contenidos de ciencias naturales, las habilidades necesarias para saber cómo abordar la pedagogía propia de la materia y sobre todo, en las actitudes que contemplan los principios éticos que deben de promoverse para despertar las vocaciones científicas y el respeto a uno mismo y a su entorno.

Resultados: La magnitud y el carácter que asumen los grandes problemas

1. Juntos por la Educación, Paraguay.

E-mail: mailnodith@gmail.com

DOI: 10.26885/rcei.foro.2017.79 
sociales, ponen en primer plano la necesidad de desarrollar actitudes y valores que orienten un comportamiento consciente acerca de las consecuencias de nuestros actos, de una actitud aceptación y de respeto hacia los demás. Entre las razones para plantear como principio la necesidad de abordar educación en valores y ciudadanía como parte fundamental de la enseñanza de las ciencias se sostiene en que esta involucra a los estudiantes en una experiencia de aprendizaje vivencial, protagónica, comprometida y consciente de su aporte a la sociedad. La formación de actitudes y valores es parte importante de la iniciación de las ciencias.

En Paraguay, según datos del Ministerio de Educación y Cultura (DGPE, 2012) existen aún grandes brechas en la formación de docentes, dado el limitado número de docentes que cuentan con la habilitación correspondiente para el nivel en el que enseñan.

Conclusiones: La crisis de la educación es sin duda, estructural, sin embargo, en lo que atañe específicamente a la enseñanza de las ciencias es absolutamente necesario plantear nuevos caminos pedagógicos, comprendiendo que un saber disciplinario no solo comprende un cúmulo de contenidos, sino que por sobre todo existen unos procedimientos didácticos propios, para enseñarlos. Procedimientos que, se definen y redefinen a la luz de las necesidades del siglo $X X I$. Lo anterior nos interpela a repensar la enseñanza de las ciencias, con un nuevo objetivo, la alfabetización científica universal y nos orienta a la necesaria pero impostergable mirada hacia implementación de un ajuste en el curriculum de la formación docente de ciencias naturales.

Se asume que la alfabetización científica es un proceso imprescindible en la formación de todo ciudadano, que impacta de forma irreversible en las decisiones que toma día a día y que impactan en el mejoramiento de su calidad de vida y de su entorno.

La adquisición de conceptos científicos fundamentales, el desarrollo de formas de pensamiento y las metodologías de trabajo propias de las ciencias, depende de los aprendizajes que se hayan logrado en los primeros años de escolaridad. Una nueva cultura docente de la educación científica - propia de un nuevo tiempo y una nueva sociedad, la sociedad del conocimiento - requiere que la transferencia de conocimiento en el aula supere el modelo reduccionista y dogmático de formación del profesorado para pasar a promover el desarrollo de competencias y habilidades cognitivo-lingüísticas en los estudiantes, facilitando el aprendizaje de las Ciencias Naturales para, el desarrollo del pensamiento crítico y creativo, la integración cultural de ciudadanos comprometidos con el desarrollo social y ambiental, así como la promoción y consolidación de los valores democráticos.

Es necesario desarrollar una educación en ciencias de calidad, relevante y sustantiva. Por tanto, para asegurar la presencia de docentes de ciencias competentes en las escuelas, debemos trabajar en aumentar el número de interesados en cursar el itinerario de formación docente de ciencias. 
Por otra parte una educación en ciencias significativa y de calidad, en el curriculum de formación docente en ciencias supone la incorporación de los siguientes aspectos elementales:

- En el eje ideológico incluir análisis históricos, sociológicos y de valores, así como otros aspectos relacionados con el rol que ocupa la ciencia en la sociedad, y la función de la educación científica en la formación de la ciudadanía.

- En el eje actitudinal incluir la práctica de las actitudes científicas y la reflexión sobre las creencias hacia la ciencia y hacia su enseñanza.

- El eje conceptual incorporar el logro de aprendizajes en aquellos conocimientos relacionados con el conocimiento pedagógico del contenido de ciencias naturales.

Las actitudes, habilidades y los conocimientos constituyen un componente fundamental de la capacidad y competencia de los docentes.

Palabras clave: alfabetización científica, didáctica, enseñanza de las ciencias, cosntructivismo.

\section{Referencias}

Adúriz Bravo, A. \& Izquierdo-Aymerich, M. (2009). Un modelo de modelo científico para la enseñanza de las ciencias naturales. Revista Electrónica de Investigación en Educación en Ciencias. Recuperado de: http://www. scielo.org.ar/pdf/reiec/nesp/nespa04.pdf

Ministerio de Educación y Cultura. Dirección General de Planificación Educativa. (2012). Capacitación de los educadores para mejoramiento de los aprendizajes de niños, niñas, jóvenes y adultos a nivel nacional. Asunción: MEC. Recuperado de: http://www.stp.gov.py/v1/?wpfb_dl=53

Näslund-Hadley, E., Martínez, E., Loera Varela, A. \& Hernández Agramonte, J. M. (2012). El camino hacia el éxito en matemáticas y ciencias: Desafíos y triunfos en Paraguay: Nuevos hallazgos del Banco Interamericano de Desarrollo sobre el pensamiento crítico en la educación pre-primaria y primaria. Washington: BID. 